\title{
The new methodological approach to division of angle, with change of dimensionality and perspective for the sake of improved efficiency, faster insight to the essence of the process and problem-solving in real time and under outdoor conditions
}

\author{
D. Knežević, and M. Laban
}

\begin{abstract}
The dividing of an angle at a certain proportion in an unambiguous way was shown by an analytical approach, taking into account the prospects and dimensionality [3]. Looking at the problem from the standpoint of antique principles and simultaneous contemporary approach to descriptive geometry proved to be necessary modernization of the tools used in solving of the practical construction problems by drawing. Importance of drawing on paper respective software tools used on PCs and tablets was emphasized. The fastest and most reliable solutions mostly were presented by simple models. The application of this methodological approach is seen in a system for monitoring remote objects [1,2], since the method allows easier and faster transformations (addition, subtraction, multiplication and division) of the values of the measured angular widths with respect to: the reference direction and the relative value of angle covers between multiple detected objects.
\end{abstract}

Keywords — division of angle, method, dimensionality.

\section{INTRODUCTION}

$\mathrm{O}^{\prime}$ UR ordinary life has progressed from the times of Archimedes, but he was the one who established many areas of science by his discoveries. He methodologically has set the model of integral calculus. Mechanics significantly advanced after discovery of the leverage, but it is still in use. Of course, progress in astronomy and in physics of fluid was enormous, but fundaments of those sciences were set by the great Greek. In this work we slightly differently perceived relations of vertical piles, related to its withdrawn generating coatings and related appropriate bases with mapped divisions

D. Knežević is student with the School of Electrical Engineering, University of Belgrade, Serbia

1) M. Laban was with the School of Electrical Engineering, University of Belgrade, Serbia. He is now with the Mathematical Institute of the Serbian Academy of Sciences and Arts, Belgrade, Serbia on their circles.

A large part of mathematics was built after Archimedes, but ruler and divider stayed basic tools. Any geometrical problem that can't be solved by construction, using only ruler and divider, is still considered unsolvable. Though practical life and necessity to solve engineering problems require introducing of new technical tools capable to solve the problems which can't be solved by a ruler and divider only. Is a drawing by a ruler and divider so precise? In descriptive geometry is not accepted to use curvimeter, which is common in orienteering, or protractor, because they are both considered imprecise. But the mere fact that one draw lines that necessarily have thickness using the ruler and divider means that the proposal to introduce a new technical aid cannot be dismissed in advance as inaccurate. Development of PCs led to applications for the drawing and presenting objects in 3D. These applications can simulate moves of ruler and divider, but their wider use is limited by copyright laws and by commercial side of their universal use in general. It can be added that the computer image that simulates a 3D view, represents a special problem [1], when something has to be implemented in technical drawing. In fact, all these 3D programs are made with a purpose that their final result be displayed on a 2D computer screen, instead of simple adjusting of the desired display on the paper.

As a rule, descriptive geometry functions in projections which are fixed, starting from the initial position. The idea here is to get into practice adjusting of these projections to the specific problem which is to be solved, so that by a series of iterations those projections be led to the desired result in line with the principle of prospective dynamization, which is in a form of a more general principle of mapping dynamization was introduced to mathematics by prof. Laban [3]. 


\section{OBSERVATION'S ATOM}

The introduction of technical equipment and tools into everyday life changes the standards of scientific observation. For example, anyone who has anything to do with photography today (either as an amateur or professional) describes its characteristics of the photos in megapixels. The number of megapixels is a kind of equivalent of quality of the photo and details on it. It is therefore necessary to introduce to science a new term (concept) which expresses the lowest possible perception of a thing or process. So, what is less of that perceptual minimum DOES NOT EXIST for the methodological approach in a scientific review, and is ignored as such.

We will call the new "OBSERVATION'S ATOM". From the geometrical point of view, observation's atom is the minimum area which corresponds to the adherence of the point in the real space, i.e. to the item which can be called "the visible point" (minimum discernible trace of pen) on the practical drawing. With digital photography, observation's atom is a pixel in the frequency domain (when we talk about music), it could be adopted by $1 \mathrm{~Hz}$ for the human hearing and so forth.

The task of the descriptive geometry is to allow easy construction of certain geometrical figures on paper, and for the needs of engineering practice in real time [1.2]. Despite the existence of modern software tools for drawing on personal computers and tablets, their application in practice is limited by many factors that greatly hamper their practical use under hard and hardest conditions (for instance during wartime). For these purposes is much more efficient and safer, so called "sketching on paper", whose correction and adjustment of the real situation in practice is much faster and easier.

\section{PERSPECTIVITY}

If we want to carry out the mapping of the object $A$ in an ndimensional space to the object $\mathrm{A}^{\prime}$, so as to apply to the $\mathrm{A}^{\prime}=$ $\mathrm{kA}$, where $\mathrm{k}$ is the unique relationship of proportionality, in the case where $\mathrm{k}$ is a constant (linear dependence) or given by the corresponding set of relationships that values for the representative points of the object $\mathrm{A}$, which is mapped, the mapping can be carried out in relations to arbitrarily selected point of perspectivity $\mathrm{O}$ within the space in which this mapping is performed.

Note a segment $\mathrm{d}_{1}$ on the object $\mathrm{A}$. If by any point $\mathrm{O}$ within the observed space, we draw the two directions, which contains the ends of segment $d_{1}$, it is obvious that can be spot segment $d_{1}$ ' which is parallel to a segment $d_{1}$ and whose ends lie on the ends of the two directions, so that it is valid $\mathrm{d}_{1}{ }^{\prime}=\mathrm{k} \mathrm{d}_{1}$. If we take spot another segment $\mathrm{d}_{2}$, it is obvious that the same applies. The rule will be effective if it is introduced $n$ segments under consideration. If we introduce the $(n+1)$ segment, the rule of mapping (copying) will be preserved, because the number of segments does not affect the law that the mapping is performed. Through this process, we proved that every point in $\mathrm{n}$-dimensional space can be mapped in an unambiguous manner from the object A to the object A ', so it is true that $\mathrm{A}^{\prime}$ $=\mathrm{kA}$ and that the principle of perspectivity does not depend on the particular choice of point of perspective within that space.

Since this mapping applies to all points of the object $A$, then certainly is true for some 3 non-collinear points belonging to him. These three points define a plane, but also a triangle in it where the points are the ends of the sides of the triangle. Thus, the observed triangle can be mapped to another, based on the principle of perspectivity. During this mapping, the metric values satisfy the relation $\mathrm{A} '=\mathrm{kA}$, if $\mathrm{k}$ is a linear constant, while the angles are mapped unambiguously $\mathrm{A}^{\prime}=\mathrm{A}$ (Fig 1).

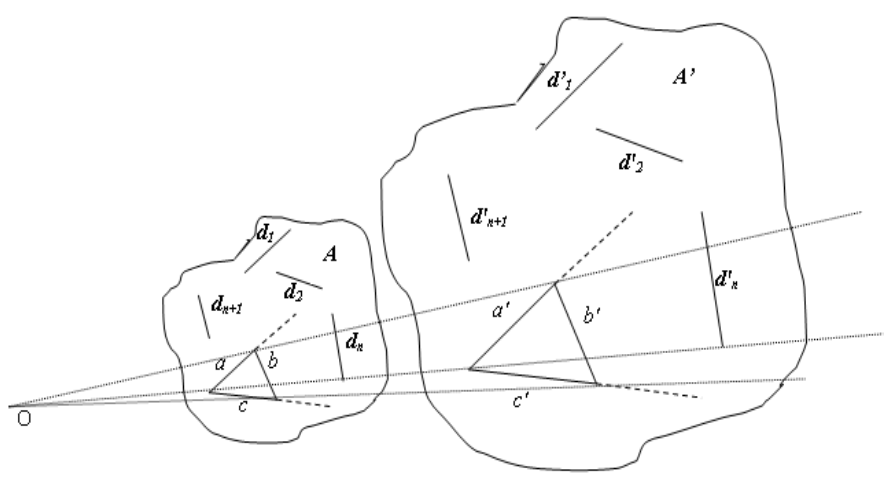

Fig. 1 An example of perspectivity

The method can be applied to mapping an endless surfaces such as the angles, but only their original values, without changing of the value of their size $\alpha^{\prime}=\alpha$.

\section{DIMENSIONALITY}

Dimensionality, as one of the three general principles of mathematical modeling, was introduced to mathematics by prof. Laban [3]. Starting from this idea, we used it to analyze our particular case, where the solution to the problem given in the two-dimensional case we get using transition of the problem by perspective in the three-dimensional case, in which form we solve it, then so obtained solution we put back by perspective to two dimensions. In this way we get a possible solution in the required two-dimensional form.

So let's try to map an attitude divide some segment, choosing an arbitrary point inside of it, on the other, a similar segment $\mathrm{d}_{1}{ }^{\prime}=\mathrm{k} \quad \mathrm{d}_{1}$. Hence, if $\mathrm{d}_{1}=\mathrm{a}_{1}+\mathrm{b}_{1}$, and then $\mathrm{d}_{1}{ }^{\prime}=\mathrm{a}_{1}{ }^{\prime}+\mathrm{b}_{1}{ }^{\prime}=\mathrm{ka}+\mathrm{kb}$.

Segment being a one-dimensional magnitude cannot be split in this way within one-dimensional space. Its divisibility is in an arbitrary relation is feasible only by introducing another dimension, that is, in two-dimensional space. The dividing segment and the segment with the entered size divisions are placed on two lines that intersect, so that an end of each of them coincides with the intersection point of their two lines. By connecting the ends of segments, which does not belong to the intersection point of two lines in which the segments are located, and by drawing the line parallel to so made segment, which passes through a point of division to the reference 
segment, on the other segment in cross-section with this new line, we receive a point corresponding to the proportional share to the segment that is dividing (proof can be carried out on the basis of the similarity of the triangles), Fig.2.
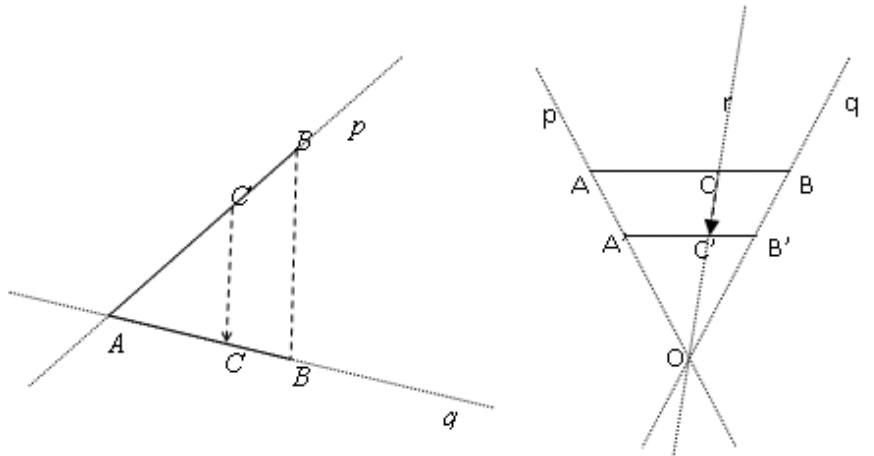

Fig. 2 An example of dividing a segment

This division like we can find out on the basis of the model of perspectivity by selecting points on the reference segment. The same applies if you had a number of points within a segment reference in a row at equidistant range, ie. along when divided into several equal parts. Evidence also follows from the principle of perspectivity and similarity of triangles.

When we divide the limited areas, which by their nature, are two-dimensional size, at the same number of parts, then the number of parts that need to be equal to the multiple of unit divisions at each dimension (multiples are natural numbers). If the request was that the area is divided in a certain number of parts, but be preserved by equality of areas, but not the coincidence of their form, it is also possible (number of divisions corresponds to the product of a natural number). If we were to divide a semi-infinite surfaces, such as angles, then we can get a division of an angle in the equal parts (the product is a natural number), or the division in the ratio (the ratio may be a real number), so that would be given containing cross-sections that pass through the point of intersection of arms angle, Fig 3 .
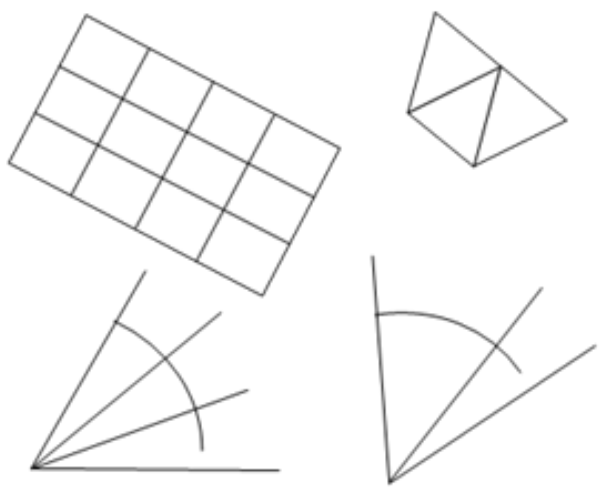

Fig. 3 Examples of surfaces divisions

When we divide the angle $\beta^{\prime}=\mathrm{k} \beta$, on the basis of distinctions of $\alpha^{\prime}=\mathrm{k} \alpha$ (angle have to be defined as a constant dependence of the ratio of the arc length of the radius to the corresponding (same) radius of the same cross-section of the lines, which are the arms of the observed angle, with arches which start on one arm, and ending at the other to define a unique center of radius at the intersection of that lines, i.e. the arms), this division can be made by cone concept. Since we start from the known division of the angle, at the angle of the constructed reference can be constructed an arc from its vertices which intersects each pair of arms and the direction that divides the angle (the angle passing through the center). If now forms a clip is limited by this arc and the arms of angle, which represents the cone envelope, it is possible in a $3 \mathrm{D}$ space, to make a circle on a basis of this cone, the points of the envelope shell and the reference point. You can easily find the center of a circle around the base reference cone inflicted divisions. Constructed on the angle that is divided, it is possible to construct an arbitrary arc, with the center of circumcenter circle located at the intersection of the arms of the angle which cuts the arms that angle. If the clip is formed limited by this arc and arms of that angle, which also constitutes an envelope of the cone, it is possible in $3 \mathrm{D}$ space, on its basis applied a circle and determines its center, Fig. 4

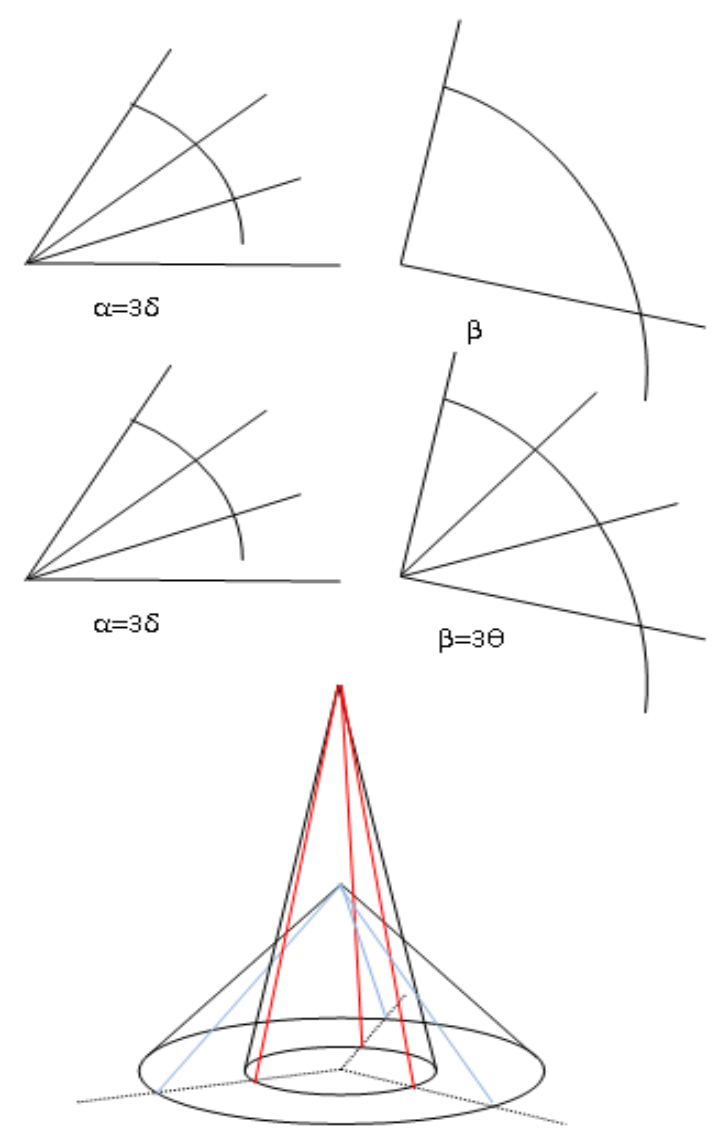

Fig. 4 Example of the dividing of the angle new methodological procedure

Since we know the radius of the circle, it is necessary to use it to construct a circle with the center corresponding to the reference center of the circle with the division. Then it should 
draw directions from the center of the reference circle through the reference points on the circle to the circle we cause division. Now we need to map in $3 \mathrm{D}$ space the reference points on the envelope, taking care that each point of the envelope shell coincide with the corresponded connecting point on the reference division circle. If this clip unfolded and re-inserted into the $2 \mathrm{D}$ space, at the arc we obtain a point through which should pass a line from the angle center which divides that angle in the parts of a given ratio. The same principle applies when the angle is divided into any number of equal parts.

\section{CONCLUSION}

The present new methodical approach is based on the known principles of perspectivity, as well as on the principles of dimensionality and dynamization, which are introduced into mathematics by Laban [3]. The using of the Laban's principle of dimensionality [3] created conditions for problem dividing angle becomes geometrically uniquely solvable (absolutely, without entering the error). From the standpoint of perspectivity the best angle of vision sought, as required when shooting in the photographic and film techniques. By applying Laban's principle of dynamization to the process of transformation from a flat surface to a curved space and vice versa process of solving problems in the illustrated case trisection angle is brought to an end.

\section{REFERENCES}

[1] D. Knežević, M. Dragović, M. Srećković, V. Ibrahimović, A.Kovačević, Numerical com-plexity of real-time tracking of objects in defined space by infrared optoelectronic devices, Indian J.Pure and Apl.Phys. Vol.52, pp.1-6, 2014.

[2] Dragan Knežević, Milesa Srećković, Saša Kočinac, Vedran Ibrahimović: " The Application Of Spatial Triangulation For Instantaneous Tracking Of Flying Objects In Specified Area", Annals Of The Faculty Of Engineering Hunedoara - Journal of Engineering, Tome V (year 2007), Fascicole 2, pp. 93-104.

[3] Prof. Dr Miloš M. Laban: „Three general principles in mathematical modeling“", monograph, Serbian official publishing company for textbooks and teaching aids, Belgrade, 2000. 